\title{
Breast cancer neoadjuvant endocrine therapy and COVID-19: a renewed breath with future perspectives
}

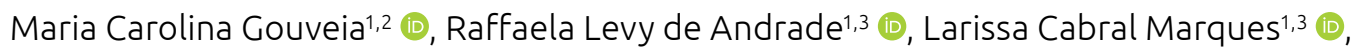 \\ Candice Amorim de Araujo Lima Santos ${ }^{4,5}$ [ , Ariani Impieri Souza 6 (1)
}

\section{ABSTRACT}

In 2020, the COVID-19 pandemic is the major healthcare concern around the world. The infection is especially severe to those with immune system suppression, including patients with cancer. In order to mitigate the negative effects of COVID-19, guidelines have been developed by societies worldwide to review oncology care during this pandemic time. Neoadjuvant endocrine therapy (NET) is a well-stablished option for hormone positive (HR) HER2 negative breast cancer and showed a positive response in breast conservative surgery with substantially less toxicity. Compared to chemotherapy, the NET cost is lower, and its administration is easier, due to less medical visits. Even with remarkable advantages, NET remains taking less place in treatments than it might have. Periods of humanity crisis, such as World Wars and other pandemics, boosted the development of science and established many treatments, which are currently practiced. New data generated during the COVID-19 outbreak can inspire more trials comparing chemotherapy to endocrine therapy within the neoadjuvant setting. The purpose of this letter is to suggest NET as a safe low toxicity treatment strategy for breast cancer, not only to postpone breast cancer surgery during the pandemic, but also to become a standard therapy, a flame kept burning crossing the COVID-19 border.

KEYWORDS: breast neoplasms; coronavirus infections; neoadjuvant therapy; anastrozole; tamoxifen.

New coronavirus disease (COVID-19), caused by SARS-CoV-2, became a major healthcare concern in 2020. On March $11^{\text {th }}$, the World Health Organization (WHO) declared the COVID-19 outbreak a global pandemic. ' Globally, as of August 2020, there have been over 23 million cases and 810,000 confirmed deaths, numbers which are certainly underestimated. ${ }^{2}$

COVID-19 is associated to different presentations, ranging from asymptomatic infections to pneumonia, severe acute respiratory syndrome, and death. Up to date, people older than 60 and/ or those with immune system suppression, including patients with cancer, are more vulnerable to infection. ${ }^{2,3}$

Cancer patients need diagnosis, evaluation, and treatment even during a pandemic. It is relevant to consider them, however, immunocompromised and at an increased risk of experiencing COVID-19-related serious events (requirement for mechanical ventilation, intensive care admission, and/or death) when compared to the general population. ${ }^{4,5}$ Guidelines intending to reduce the negative effects of COVID-19 have been developed by oncology societies around the world. ${ }^{6,7}$

The Centers for Disease Control and Prevention and the American College of Surgeons have proposed to postpone elective surgeries, if possible. Evidence suggests that patients who received surgery and concomitantly contracted COVID-19 were at much higher risk of incurring severe clinical events than those who did not have surgery. ${ }^{6,7}$ These new protocols have emphasized the importance of carefully selecting patients eligible for surgical procedures during this time. ${ }^{6}$

The decision of postponing cancer surgeries should be dimensioned with the possibility of disease progression, as well as faceto-face meetings to chemotherapy infusion or follow up consultations. COVID-19-free departments are an attractive alternative to provide greater safety for patients and staff, but their implementation takes time and is still an operational challenge. ${ }^{6,7}$

${ }^{1}$ Breast Surgery, Instituto de Medicina Integral Professor Fernando Figueira - Recife (PE), Brazil.

${ }^{2}$ Grupo Oncoclínicas - Recife (PE), Brazil.

${ }^{3}$ Real Instituto de Mastologia/Real Hospital Português - Recife (PE), Brazil.

${ }^{4}$ Clinical Oncology, Instituto de Medicina Integral Professor Fernando Figueira - Recife (PE), Brazil.

${ }^{5}$ Clinical Oncology, Rede D'Or - Recife (PE), Brazil.

${ }^{6}$ Gynecology, Instituto de Medicina Integral Professor Fernando Figueira - Recife (PE), Brazil.

*Corresponding author: raffaelandrade@hotmail.com

Conflict of interests: nothing to declare.

Received on: 06/29/2020. Accepted on: 08/31/2020. 
Given the worldwide magnitude of breast cancer in terms of public health, reviewing care strategies is vital. With this article, we intend to present some data to review and encourage the use of neoadjuvant hormone-therapy during the COVID-19 crisis and after it.

During this viral outbreak, neoadjuvant therapies have been used to provide an opportunity for safely postponing breast cancer surgery. ${ }^{8}$ If equivalent, approved oral therapy regimens are recommended instead of parenteral chemotherapy to reduce patients risk of exposure to the virus without compromising oncological outcomes. ${ }^{5,7}$

Neoadjuvant endocrine therapy (NET) is a low-toxicity approach to hormone receptor positive (HR) and HER2 negative breast cancer. NET effectiveness is equivalent to chemotherapy in downsizing tumors, raising breast conserving surgery in patients with $\mathrm{HR}$ positive disease, and providing information on endocrine responsiveness. ${ }^{910}$ Several studies showed that monotherapy with aromatase inhibitors had a similar clinical, radiological, and breast conserving surgery rates than neoadjuvant standard chemotherapy, and both treatments had a low pathologic complete response (pCR) rate of roughly $10 \% .910$

However, whereas neoadjuvant chemotherapy (NCT) is a common strategy, NET is underutilized, either due to an uncommon pathologic complete response after neoadjuvant endocrine therapyexpected for luminal breast tumors-or to the less useful prognostic information after treatment. ${ }^{10,11}$ A recent study comparing NET versus NCT found $11 \%$ of nodal pCR in previous node-positive breast cancer treated with NET (not statistically different from NCT group), and $77 \%$ woman became eligible for breast conserving surgery (BCS). ${ }^{12}$ NET is more likely to be successful in de-escalating breast surgery than axillary surgery, because pathologic complete response is not necessary to allow downstaging to BCS, but it is required to avoid axillary lymph node dissection after neoadjuvant treatments. ${ }^{12}$

NET is usually limited to the treatment of elderly postmenopausal women with large tumors, who were the worst candidates for NCT or upfront surgery. An examination of the United States National Cancer Data Base reveals that only $3 \%$ of potentially eligible patients received NET. ${ }^{10,11,13}$ Evidence shows various benefits in the use of NET for a wider spectrum of patients (younger, including premenopause, and potentially candidates for up-front surgery) ${ }^{10,11,13}$

Regardless of the lack of $\mathrm{pCR}$, NET results suggest that other primary endpoints should be considered. Until today, there was no evidence of an increased overall survival (OS) in patients who achieve pCR for HR positive tumors. The Preoperative Endocrine Prognostic Index (PEPI)-an index that combines, among other clinicopathologic factors, the residual Ki67 score and measurement of estrogen receptors during NET-has also found an application in clinical trials and NET as a potential endpoint. The idea of assessing clinical and biomarker responses has inspired the development of novel clinical trial designs for measuring the impact of endocrine agents. ${ }^{7-9}$ Besides that, another motivating outcome is the number of patients who initially would have undergone radical surgeries and were converted into conservative breast surgeries after NET. ${ }^{14}$
Several advantages of NET can be especially valuable during a pandemic. When compared to anthracycline and taxane-based chemotherapy, NET has significantly lower toxicity. Some randomized trials comparing NET and NCT showed equivalent response and rate of breast conservative surgery for both treatments, with substantially less severe adverse events, such as neutropenia, febrile neutropenia, and cardiotoxicity in endocrine treatment. ${ }^{13,15}$

Endocrine therapy offers a lower cost, easier medication administration and, obviously, less visits to health units, not only to receive medication, but less hospitalizations due to side effects. All these factors, added to the safety and effectiveness of this type of neoadjuvant therapy, highlights NET as an excellent treatment option during the COVID-19 pandemic. Additionally,NET can show its value in the window trial space as treatment strategies for CDK4/6inhibitors, and other new drugs and protocols are developed and continue to evolve..$^{15-19}$

The present historical moment is helping the scientific community to rethink current practices, in which most positive HR breast cancer patients candidates to neoadjuvant therapy still receive chemotherapy, even experiencing less robust responses when compared to other molecular profiled tumors. ${ }^{15,19}$

The COVID-19 pandemic, just as other difficult periods of humanity, has not only changed daily routines, but also forced specialists to replan management options of cancer patients in these new times. During periods of crisis, generating opportune evidence for treatment options for cancer patients is vital for the community to identify best practices and optimized treatment plans for those susceptible to the virus. The higher risk of COVID-19-related complications for patients with cancer expresses the need for creating pragmatic approaches, and a deep review of potential available treatments.

Surgical entities suggest increasing the criteria for selecting candidates for up-front surgery. NET for luminal breast cancer can perform an important role in this unprecedented pandemic scenario for our generation; it combines a safe oncological outcome with less toxicity and exposure to the current unfavorable hospital environment. A rising number of patients undergoing NET is foreseen due to this current pandemic.

New data generated during the COVID-19 outbreak can inspire further research and trials to compare chemotherapy to endocrine therapy on the neoadjuvant scenario. More than ever, personalized medicine is the current goal to keep patients safe and healthy.

\section{AUTHORS' CONTRIBUTIONS}

M.G.: Conceptualization, project administration, supervision, validation, visualization, investigation, methodology, writing - review \& editing.

R.A.: Project administration, supervision, validation, visualization, investigation, methodology, writing - review \& editing.

L.M.: Writing - review \& editing, formal analysis.

C.S.: Formal analysis, review \& editing.

A.S.: Formal analysis, review \& editing. 


\section{REFERENCES}

1. World Health Organization. Director General's opening remarks at the media briefing on COVID-19 [Internet]. World Health Organization; 2020 [accessed on May 26, 2020]. Available at: https:// www.who.int/dg/speeches/detail/who-director-general-s-openingremarks-at-the-media-briefing-on-covid-19---11-march-2020

2. Wu Z, McGoogan JM. Characteristics of and Important Lessons from the Coronavirus Disease 2019 (COVID-19) Outbreak in China: Summary of a Report of 72314 Cases from the Chinese Center for Disease Control and Prevention. JAMA. 2020;323(13):1239-42. https://doi.org/10.1001/jama.2020.2648

3. Sidaway P. COVID-19 and cancer: what we know so far. Nat Rev Clin Oncol. 2020;17:336. https://doi.org/10.1038/s41571-020-0366-2

4. Yu J, Ouyang W, Chua MLK, Xie C. SARS-CoV-2 Transmission in Patients with Cancer at a Tertiary Care Hospital in Wuhan, China. JAMA Oncol. 2020;6(7):1108-10. https://doi.org/10.1001/ jamaoncol.2020.0980

5. Gosain R, Abdou Y, Singh A, Rana N, Puzanov I, Ernstoff MS. COVID-19 and Cancer: a Comprehensive Review. Curr Oncol Rep. 2020;22(5):53. https://doi.org/10.1007/s11912-020-00934-7

6. American College of Surgeons. COVID 19: Elective Case Triage Guidelines for Surgical Care 2020 [Internet]. 2020 [cited on May 15, 2020]. Available at: https://www.facs.org/covid-19/ clinical-guidance/elective-case/breast-cancer

7. Curigliano G, Cardoso MJ, Poortmans P, Gentilini O, Pravettoni G, Mazzocco K, et al. Recommendations for triage, prioritization and treatment of breast cancer patients during the COVID-19 pandemic. Breast. 2020;52:8-16. https://doi. org/10.1016/j.breast.2020.04.006

8. Marti C, Sánchez-Méndez JI. Neoadjuvant endocrine therapy for luminal breast cancer treatment: a first-choice alternative in times of crisis, such as the COVID-19 pandemic. Ecancer. 2020;14:1027. https://doi.org/10.3332/ecancer.2020.1027

9. Palmieri C, Cleator S, Kilburn LS, Kim SB, Ahn SH, Beresford M, et al. NEOCENT: a randomised feasibility and translational study comparing neoadjuvant endocrine therapy with chemotherapy in ER-rich postmenopausal primary breast cancer. Breast Cancer Res Treat. 2014;148(3):581-90. https://doi.org/10.1007/s10549-014-3183-4

10. Spring LM, Gupta A, Reynolds KL, Gadd MA, Ellisen LW, Isakoff SJ, et al. Neoadjuvant endocrine therapy for estrogen receptor-positive breast cancer a systematic review and meta-Analysis. JAMA Oncol. 2016;2(11):1477-86. https://doi.org/10.1001/jamaoncol.2016.1897
11. Charehbili A, Fontein DBY, Kroep JR, Liefers GJ, Mieog JSD, Nortier JWR, et al. Neoadjuvant hormonal therapy for endocrine sensitive breast cancer: A systematic review. Cancer Treat Rev. 2014;40(1):86-92. https://doi.org/10.1016/j. ctrv.2013.06.001

12. Montagna G, Sevilimedu V, Fornier M, Jhaveri K, Morrow M, Pilewskie ML. How Effective is Neoadjuvant Endocrine Therapy (NET) in Downstaging the Axilla and Achieving Breast-Conserving Surgery? Ann Surg Oncol. 2020. https://doi. org/10.1245/s10434-020-08888-7

13. Chiba A, Hoskin TL, Heins CN, Hunt KK, Habermann EB, Boughey JC. Trends in Neoadjuvant Endocrine Therapy Use and Impact on Rates of Breast Conservation in Hormone Receptor-Positive Breast Cancer: A National Cancer Data Base Study. Ann Surg Oncol. 2017;24(2):418-24. https://doi. org/10.1245/s10434-016-5585-5

14. Guerrero-Zotano AL, Arteaga CL. Neoadjuvant trials in ER+ breast cancer: A tool for acceleration of drug development and discovery. Cancer Discov. 2017;7(6):561-74. https://doi. org/10.1158/2159-8290.CD-17-0228

15. Murphy CG. The Role of CDK4/6 Inhibitors in Breast Cancer. Curr Treat Options Oncol. 2019;20(6):52. https://doi. org/10.1007/s11864-019-0651-4

16. Wang Y, He L, Song Y, Wu Q, Wang H, Zhang B, et al. The tumour response of postmenopausal hormone receptor-positive breast cancers undergoing different types of neoadjuvant therapy: A meta-analysis. BMC Womens Health. 2020;20(1):17. https://doi. org/10.1186/s12905-020-0879-y

17. Dietz JR, Moran MS, Isakoff SJ, Kurtzman SH, Willey SC, Burstein $\mathrm{HJ}$, et al. Recommendations for prioritization, treatment, and triage of breast cancer patients during the COVID-19 pandemic. the COVID-19 pandemic breast cancer consortium. Breast Cancer Res Treat. 2020;181(3):487-97. https://doi.org/10.1007/s10549-020-05644-z

18. American Society of Breast Surgeons. ASBrS Resource Guide to Endocrine Therapy in the COVID-19 Pandemic. American Society of Breast Surgeons; 2020.

19. Curigliano G, Gómez Pardo P, Meric-Bernstam F, Conte P, LolkemaMP,BeckJT, etal.Ribociclib plus letrozolein earlybreast cancer: A presurgical, window-of-opportunity study. Breast. 2016;28:191-8. https://doi.org/10.1016/j.breast.2016.06.008 